\title{
Answer to the Letter to the Editor of J. Padulo et al. concerning "Range of motion of thoracic spine in sagittal plane" by D. Morita et al. Eur Spine J (2014) 23:673-678
}

\author{
Daigo Morita
}

Received: 26 April 2014/Revised: 26 April 2014/Accepted: 26 April 2014/Published online: 15 May 2014

(c) Springer-Verlag Berlin Heidelberg 2014

We thank Dr. J. Padulo et al. for addressing our article. We wish to respond to their questions as follows:

1. Certainly, we have not reported the strict dynamic alignment of the thoracic spine. Our study has shown the segmental kyphotic angle and range of motion within the normal functional motion of the thoracic spine. Furthermore, we have not assessed torque, from minimum to maximum, over flexion and/or extension angle, over rotation time, as we have mentioned partially in the limitations of our study. We will investigate these points in our future study.

2. Although triangular pillows (bending spines) have been of the same size as you pointed out, we have tried to get the maximum flexion and extension positions of each patient using towels according to each physical constitution. Different positions could be obtained by different physical constitutions. However, we have not assessed the body height, body (trunk) lengths, and body mass. We will investigate these points in our future study.

3. Environmental conditions and subject's placement in CT performing have not been assessed. Although intra- class correlation coefficient has not been also assessed, all parameters were measured once on two different days by a spinal surgeon, and average values were adopted.

4. We could obtain almost the same results using Student's $t$ test but not the Mann-Whitney test. In our study, we have used the Mann-Whitney test according to reviewer's indication. As you pointed out, standard deviation was bigger than mean in some cases. However, we have shown the results as obtained.

5. In Figs. 3, 4, and 5, mean data have been shown without any standard deviation or standard error, as you pointed out.

We have proceeded our study as much as possible in the environment, which has been restricted in clinical aspects, as described in limitations. We also think that it is ideal if we can realize all as you pointed out.

Conflict of interest None.
D. Morita $(\square)$

Department of Orthopaedic Surgery, Hamamatsu Medical

Center, 328 Tomitsuka-cho, Naka-ku, Hamamatsu,

Shizuoka 432-8580, Japan

e-mail: daigood0301@yahoo.co.jp 